\title{
Popliteal Artery Injury: Short Term Outcome With or Without Skeletal Trauma
}

\author{
MD. SAIF ULLAH KHAN ${ }^{1}$, SANURULALAM ${ }^{2}$, NASIR UDDIN AHMED ${ }^{3}$ \\ ${ }^{1}$ Department of Vascular Surgery, Bangabandhu Sheikh Mujib Medical University, Dhaka, ${ }^{2}$ Department of Vascular Surgery, National \\ Institute of Cardiovascular Diseases, Dhaka, ${ }^{3}$ Department of Cardiac Surgery, National Institute of Cardiovascular Diseases, Dhaka \\ Address for correspondence: Md. Saif Ullah Khan, Department of Vascular surgery, Bangabandhu Sheikh mujib Medical \\ University, Dhaka
}

\begin{abstract}
Short term outcome of popliteal artery injury with or without skeletal trauma was studied during the period from January 2005 to December 2005, in the department of cardiovascular surgery, National Institute of Cardiovascular Diseases, Dhaka, Bangladesh. Thirty patients undergoing surgical management for popliteal artery injury were divided equally into two groups, 15 patients with skeletal trauma (Group A) and 15 patients without skeletal trauma (Group B). Study shows that seven patients had postoperative complications in entire group, 6 in group A and one in group B. Postoperative infection was the most common complication (16.7\% of total patients) in both groups accounting for 4 (26.6\% within group) patients in group $A$ and in one patient (6.7\% within group) in group B respectively. Limb amputation is the most important parameter of outcome, which was needed in two patients (13.3\% within group) of group A and in none of group B. Other complications were thrombosis, neurological deficit and absent distal pulse, all of which occurred exclusively in group A.
\end{abstract}

Keywords: Popliteal artery, Injury, Outcome

\section{Introduction}

Approximately 90\% of all peripheral arterial injuries occur in an extremity. ${ }^{1}$ Injury to the popliteal artery is the most common cause of amputation in injured extremities. ${ }^{2}$ Despite technical improvements in management of popliteal artery injury, a high amputation rate is still seen, especially in patients with one or more of the following factors: extensive soft tissue injury, associated skeletal trauma, knee dislocation and prolonged ischemic time. ${ }^{3}$ The magnitude of skeletal and soft tissue injury alone or in combination was also strongly associated with an increased morbidity. ${ }^{4}$ Amputation rates approaching 70\% still are reported from combined arterial and skeletal extremity trauma, while less than 5\% of limbs currently are lost following isolated arterial or skeletal trauma. ${ }^{5}$ During World War II extremity arterial injuries were routinely ligated. For popliteal arterial injury the amputation rate was 73\%. Continuing refinements in arterial surgery over the ensuing three decades have reduced limb loss in most civilian series to less than 10 to 15 percent. $^{6}$

$10 \%$ to $70 \%$ of all extremity arterial injuries are associated with skeletal trauma. Appropriate prioritization of the management of the vascular and skeletal injuries is a major determinant of limb salvage. The consensus of authorities now favors limb revascularization as the first priority in all combined extremity trauma. ${ }^{5}$

\section{Patients \& Methods}

This is a prospective observational study, carried out on patients with popliteal arterial injury who underwent reconstructive vascular surgery during January 2005 to
December 2005 in National Institute of Cardiovascular Diseases (NICVD), Dhaka, Bangladesh. Thirty patients were selected and divided equally into two groups, 15 patients of popliteal arterial injury with associated skeletal injury (Group A) and 15 patients of popliteal arterial injury without associated skeletal injury (Group B).

Detailed history of each patient under study was recorded, with special attention to the mode, nature and extent of trauma. Important and relevant data were collected preoperatively, during operative period, postoperatively, during follow up in one month period after operation and recorded.

\section{Results}

Predominant cause of vascular injury in the study was traffic accident, which accounted for vascular injury in 16 (53.35\%) patients. The other causes of vascular injury were stab, gunshot, assault by blunt weapon, sharp object and crush injury.

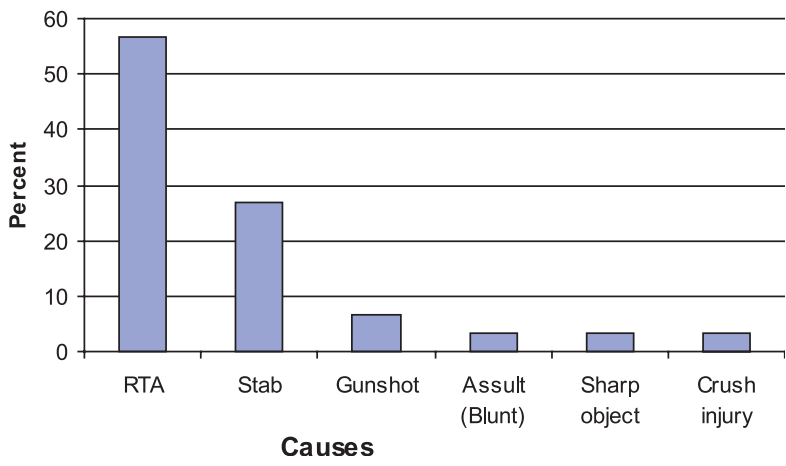

Fig.-I: Causes of vascular injury 
Common clinical finding associated with vascular injury was absence of distal pulse, pallor and poikilthermia (coldness), which were found in 28(93.3\%) patients. Presence of enlarging hematoma was found in 3(20\%) patients of group A and 8 (56.7\%) patients of group B. Two patients in group B and none in group A had pulsatile hematoma.

Table - I

Preoperative Clinical findings of vascular injuries

\begin{tabular}{lllll}
\hline Clinical sign & Finding & Total Group & Group A & Group B \\
\hline Pallor & Present & 28(93.3\%) & $14(93.3 \%)$ & $14(93.3 \%)$ \\
Distal Pulse & Present & 1(03.3\%) & $0(0.00 \%)$ & $1(06.7)$ \\
& Absent & 28(93.3\%) & 14(93.3\%) & $14(93.3 \%)$ \\
& Equivocal & $1(03.3 \%)$ & $1(06.7 \%)$ & $0(0.00 \%)$ \\
Poikilthermia & Present & 28(93.3\%) & 14(93.3\%) & $14(93.3 \%)$ \\
Capillary filling & Normal & 1(03.3\%) & 0(0.00\%) & $1(06.7 \%)$ \\
time & Delayed & 29(97.6\%) & $15(100 \%)$ & $14(93.3 \%)$ \\
Hemorrhage & Present & $1218(60.0 \%)$ & $12(80.0 \%)$ & $6(40.0 \%)$ \\
Enlarging & Present & 10(33.3\%) & $3(20.0 \%)$ & $8(56.7 \%)$ \\
hematoma & & & & \\
Pulsatile & Present & $2(06.7 \%)$ & $0(0.00 \%)$ & $2(13.3 \%)$ \\
hematoma & & & & \\
\hline
\end{tabular}

Other associated injuries were, popliteal vein injury in 10 (33.3\%) patients, muscle injury in 20 (66.7\%) patients, nerve injury in 4 (13.3\%) patients and tendon injury in 2 (6.7\%) patients.

Table-II

Associated injuries of the limb

\begin{tabular}{llll}
\hline Associated Injury & Total patients & Group A & Group B \\
\hline Bone fracture & $15(50 \%)$ & $15(100 \%)$ & $0(0.0 \%)$ \\
Popliteal vein injury & $10(33.3 \%)$ & $6(40 \%)$ & $4(26.7 \%)$ \\
Muscle injury & $20(66.7 \%)$ & $11(73,3 \%)$ & $9(60 \%)$ \\
Tendon injury & $2(6.7 \%)$ & $2(13.3 \%)$ & $0(0.0 \%)$ \\
Nerve injury & $4(13.3 \%)$ & $2(13.3 \%)$ & $2(13.3 \%)$ \\
\hline
\end{tabular}

Reconstruction of injured artery by autogenous interpositional venous graft was done in $8(26.7 \%)$ patients in group A and 1 (3.33\%) patient in group B, end to end anastomosis in 5(33.3\%) patients in group A and 7(46.7\%) patients in group B and lateral suture repair in 2(6.7\%) patients in group A and 71 (46.7\%) patients in group B .

\section{Table-III}

Type of reconstruction done for revascularization of the limb

\begin{tabular}{lccr}
\hline Type of reconstruction & Total patients & Group A & Group B \\
\hline RIVG & $9(30 \%)$ & $8(53.4 \%)$ & $1(6.7 \%)$ \\
End-to end anastomosis & $12(40 \%)$ & $5(33.3 \%)$ & $7(46.7 \%)$ \\
Lateral suture repair & $9(30 \%)$ & $2(13.3 \%)$ & $7(46.7 \%)$ \\
\hline
\end{tabular}

(RIVG- Reverse Inter-positional Venous Graft)
Postoperative infection after vascular reconstruction was the most common complication in both groups, accounting for 5 and 4 cases in group A and group B respectively. Limb amputation is the most important parameter of outcome, which was needed in 2 (13.3\% within group) patients in group A. Other complications were thrombosis of the graft/artery, neurological deficit and absent distal pulse, all of which occurred exclusively in group A.

Table-VII

Frequency distribution of postoperative complications

\begin{tabular}{llll}
\hline Postoperative complication & $\begin{array}{l}\text { Total } \\
\text { patients }\end{array}$ & Group A & Group B \\
\hline Infection & $5(16.7 \%)$ & $4(26.7 \%)$ & $1(6.7)$ \\
Thrombosis & $2(6.7 \%)$ & $2(13.3 \%)$ & 0 \\
Absent pulse distal to injury & $2(6.7 \%)$ & $2(13.3 \%)$ & 0 \\
Neurological deficit & $4(13.3 \%)$ & $4(26.7 \%)$ & 0 \\
Amputation of affected limb & $2(6.7 \%)$ & $2(13.3 \%)$ & 0 \\
\hline
\end{tabular}

\section{Discussion}

Proper management of trauma cases, especially those that require multidisciplinary approach under a single roof, is still unavailable in our country. The assessment and treatment of a patient with an extremity injury from the point of view of a possible vascular injury is often obvious, but also may be difficult. Those patients who present with "hard signs" of vascular injury like; arterial bleeding, enlarging hematoma, bruits, thrills, or pulseless ischemic limb, do not represent a diagnostic dilemma. ${ }^{7}$ This is a prospective observational study, which shows that out of 30 patients 18 (60\%) had penetrating injuries and 12 (40\%) had blunt trauma. Among the patients with skeletal injury 8 had fracture of femur, 9 had fracture of tibia and 9 had fracture of fibula singly or in combinations. No patient had joint dislocation. Muscle injury was the most common associated injury, found in 20 (66.7\%) patients. Popliteal vein was injured in 10 (33.3\%) patients and nerve was involved in 4 (13.3\%) patients.

In the Study by Lim et al., mean time from injury to the start of surgery was 12 hours. ${ }^{2}$ In the present study, The mean time laps between vascular injury and time of vascular reconstruction was 11.35 hours with $\mathrm{SE} \pm 1.32$ in Group $\mathrm{A}$ and 11.98 hours with SE \pm 1.34 in Group B. Minimum and maximum time laps (Ischemic time) being 5.5 hours and 24.3 hours respectively. Time delay between the study groups is not significant $(\mathrm{p}=.74)$. This considerable delay in onset of definitive therapy is partly due to transportation of the patient to the center. The other factors that 
contributed to this prolong ischemic time include delayed recognition of vascular injury at the primary treatment facility, treatment of other injuries like stabilization of associated skeletal fractures in a different center, and resuscitation of patients in shock.

In 2003, Mohammadzade and Akbar (2003) in 145 cases of popliteal artery injury, vascular reconstructions were done by vein graft interposition in 101, primary re-anastonmosis in 28, prosthetic graft interposition in six, lateral suture in12, vein patch in five, and ligation in one. ${ }^{8}$ They reported graft occlusion in 11(7.58\%), muscle necrosis in 9(6.21\%), l sepsis in 4(2.75\%) and foot drop in $1(0.67 \%)$ patients. In the present study, vascular reconstructions were done by end-to-end anastomosis in 12 (40.0\%) cases, reverse interpositional venous grafts (RIVG) in 9(30\%) cases and lateral suture repair in $9(30 \%)$ cases. In the present study the rate of infection was the commonest postoperative complication, occurred in 5 patients (16.7\%) in the entire group. It was much higher (26.7\% within group, $n=4)$ in group A (patients with skeletal injuries), compared to (6.7\% within group, $n=1$ ) in group $B$ (patients without skeletal injury). Neurological deficit (26\% within group, $n=4$ ) and thrombosis (13.3\%within group, $n=2$ ) were present only in group A patients. Among the patients included in this study, there was no death.

All the above-mentioned complications and morbidity factors which occurred in a disproportionately higher percentage in group A (patients with associated skeletal injury) suggests poorer outcome of popliteal artery injury in patients with associated skeletal trauma.

\section{Conclusions}

It may be concluded that preoperative morbidity is higher in patients with associated skeletal trauma and their postoperative outcome is also poorer, Limb salvage is higher and morbidity is less in patients with popliteal artery injury without skeletal injury. It may also be concluded that postoperative infection should be controlled rigorously. Combined arterial and skeletal extremity trauma imparts a substantially higher risk of limb loss and limb morbidity than do isolated skeletal and arterial injuries.

We recommend that limb revascularization should be considered as the first priority in all combined extremity trauma.

\section{References}

1. Rich NM, Baugh JA, Hughes CW. Popliteal artery injuries in Vietnam. Am J Surg 1969; 118: 531-34.

2. Lim LT, Michuda MS, Flanigan DP, Pankovich A. Popliteal artery trauma, 31 consecutive cases without amputation. American Medical Association. Available at $<$ http:// www.google.com. May 2005.

3. Yahya, Marzi M, Mwipatayi, et al. Popliteal Artery Injury: Royal Perth Experience and Literature Review.[On line], Blacwell Publishing ltd [7 October 2005].

4. Pretre R, Bruschweiler I, Rossier J. et al. Lower Limb Trauma With Injury to the Popliteal Vessels. The journal of Trauma Injury, Infection and Critical Care 1996; 40(4): 595-60.

5. Frykberg ER. Combined arterial \& skeletal extremity trauma, Trauma Wiki. Available at beta.trauma.org/traumawiki. May 2005.

6. Hood DB, Yellin AE, Weaver FA. Vascular trauma, Current Diagnosis \& Treatment in Vascular Surgery .Englewood Cliffs, N.J.:Prentice Hall. 1995;405-28.

7. Gates, Penetrating wounds of the extremities- Method of identifying arterial injury. Orthop Rev 1994;23:10-12.

8. Mohammadazade MA, Akbar MH. Presenting Features And Surgical Outcome Of Popliteal Artery Trauma In A Civilian Set-Up. Acta Medica Iranica, Available at http://www.diglib tums.ac.ir/pub/med.aspp [FEBRUARY 2005]. 